\title{
Dedication to Nathan Isgur
}

\author{
Mark B. Wise \\ California Institute of Technology, 452-48 Caltech, Pasadena CA 91125, USA
}

Nathan passed away in July after a lengthy illness. I am sure most of you are familiar with his many contributions to heavy quark physics and it is certainly fitting that we take a few minutes to honor him at the beginning of this meeting. Actually Nathan's main physics interest was the strong interactions rather than heavy quark physics per se. He was already very well known for work he did with Gabriel Karl and others on the nonrelativistic quark model before the work that he did on heavy quark symmetry and its applications. However, Nathan understood the limitations of the nonrelativistic quark model, and was thrilled that the methods he helped develop allowed one to derive systematically from the theory of the strong interactions many properties of hadrons that contain a heavy quark.

Since many of you may not be familiar with Nathan's work outside of the area of heavy quark physics, I give below a list of some of his most important non heavy quark physics papers. They are not in any particular order and might not even be the most cited. But I think they are at least some of the ones he was particularly proud of:

- "Why the pseudoscalar-meson mixing angle is $-10^{\circ}$ ", Phys. Rev. D12, 3770 (1975).

In this paper Nathan gave a simple explanation based on quark model mixing for the observed $\eta-\eta^{\prime}$ mixing angle. It is a beautiful piece of simple physics that still forms the basis of our understanding for the value of this angle.

- "P-wave baryons in the quark model", (with G. Karl) Phys. Rev. D18, 4187 (1978); "Positive-parity excited baryons in a quark model with hyperfine interactions", (with G. Karl) Phys. Rev. D19, 2653 (1979).

These two papers form the basis of the Isgur-Karl quark model for baryons. It is shocking how many properties of the baryons this nonrelativistic quark model approach is able to explain.

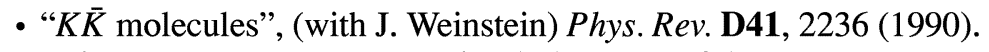

This paper gave some very simple but powerful arguments that what used to be called the $\delta(980)$ should be interpreted not as the usual type of quark model state but rather as a weakly bound kaon-anti-kaon state.

- "Perturbative QCD in Exclusive Processes", (with C. Lewellyn Smith) Phys. Lett. B217, 535 (1989).

Here Nathan argues that the perturbative predictions for exclusive processes made by Brodsky and Lepage should not be a good approximation until very large $Q^{2}$.

CP618, Heavy Flavor Physics: Ninth International Symposium, edited by A. Ryd and F. C. Porter (C) 2002 American Institute of Physics 0-7354-0064-4/02/\$19.00 
This was a very important paper because at the time it was written much of the community thought that these predictions are relevant at quite low $Q^{2}$.

- "Flux-tube model for hadrons in QCD", (with J. Paton) Phys. Rev. D31, 2910 (1985).

There are many properties of low energy QCD that cannot be described with the non relativistic quark model, for example, the properties of exotic mesons. Also one would like to have a deeper understanding of why the nonrelativistic quark model works at all. Nathan made a start on this problem in this paper.

When I called my wife Jackie from Rome to tell her that Nathan passed away she was saddened that as she put it "such a good and gentle man" had left us. I never heard Nathan raise his voice or get angry during any discussion, physics or otherwise. It's not that he could not be critical, but he was always composed and never felt the need to make someone feel small if they had not appreciated the proper reasoning in a particular argument. But still I have a hard time viewing Nathan as "gentle". I think this is because most of my interactions with him centered on physics. He was fiercely driven to understand the physics issues that he was puzzling over, and he could perform even the most bruising calculations if they would help him gain the intuition he was after. In physics being a great calculator is a little like being a great athlete, it takes discipline and perseverance as well as natural talent. Just as it is hard to picture professional athletes as gentle souls when they are off the field it is sometimes hard for me to see Nathan in that light.

I first met Nathan when I was a sophomore at the University of Toronto. He had recently come to Toronto as a graduate student and was teaching an undergraduate seminar class on statistical mechanics. He chose as the book Kittel's thermal physics, which is one of great undergraduate texts. It was a marvelous course. Nathan was a gifted teacher both in the seminar style of that course and in the more conventional lecture style as well.

Canadians are not as daring as Americans. Like most of my friends I lived at home during my undergraduate years and was more than a little nervous about leaving home to do graduate work in the United States. By the time I was looking into graduate schools Nathan was a professor at the University of Toronto. He told me that he would be happy to have me as his graduate student but insisted the best thing for me was to go to the US and get my $\mathrm{PhD}$ there.

The first time I tried, I didn't get into any of the US graduate schools that I wanted to attend, so I stayed at University of Toronto for a masters. It ended up being one of the best moves of my career. I took a graduate course on the quark model from Nathan and he also supervised my reading of Bjorken and Drell Volume 1. I have often thought what Nathan taught me that year gave me an advantage over many of my contemporaries who had more experience with tools from field theory but lacked some of the intuition that came from the type of quark model calculations that Nathan had exposed me to.

After that time I would work with Nathan whenever I came home to visit my family during the summer. Usually it was a two week visit and I would go into University of Toronto to talk with Nathan almost every weekday. That's how our collaboration on heavy quark physics started. Actually, it might have started off with a phone call before 
a visit home, but certainly much of the serious work was done during my visits home. I remember vividly that the idea for the paper where we treated semileptonic $B$ to $D$ and $D^{(*)}$ decays away from zero recoil came on the last day of one of my visits to Toronto. I only had a few hours before my flight left. The idea came to us while we were in front of the blackboard. Time was so short I didn't have time to use the restroom before heading out into traffic to get to the airport. It was a rather uncomfortable drive but I did make the flight and satisfy my other needs at the airport.

I have very fond memories of the work we did together on heavy quark physics. Intellectually it was the most exciting period of my career in physics. Even after the early 1990's and well into the period when Nathan was not well we continued to talk about particle physics. He never lost his passion for the subject and continued to publish important work. Few particle theorists have been as influential as Nathan and so universally well liked. I miss him and think about him often, as I am sure many of you also do. 